\title{
Deep Inspiration Breath Hold Reduces Dose to the Left Ventricle and Proximal Left Anterior Descending Artery during Radiotherapy for Left-Sided Breast Cancers
}

\author{
Lesley A. Jarvis ${ }^{1}$, Peter G. Maxim², Kathleen C. Horst ${ }^{2 *}$ \\ ${ }^{1}$ Department of Radiation Oncology, Dartmouth-Hitchcock Medical Center, Lebanon, USA; ${ }^{2}$ Department of Radiation Oncology, \\ Stanford University Medical Center, Stanford, USA. \\ Email: *kateh@stanford.edu
}

Received June 20 ${ }^{\text {th }}$, 2012; revised July 23 ${ }^{\text {rd }}, 2012$; accepted August $4^{\text {th }}, 2012$

\begin{abstract}
The purpose of this study was to analyze motion of the left anterior descending coronary artery (LAD) and left ventricle during normal breathing and deep inspiration breath hold (DIBH). This is a dosimetric study utilizing free-breathing and static DIBH scans from eleven patients treated with radiotherapy for breast cancer. The anterior-posterior displacement along the length of the LAD was measured in each respiratory phase. Standard treatment plans targeting the whole breast without treatment of the internal mammary lymph nodes were generated and dose to the LAD and LV calculated. Non-uniform movement of the LAD during respiratory maneuvers with the proximal third exhibiting the greatest displacement was observed. In DIBH compared to end-expiration (EP), the mean posterior displacement of the proximal $1 / 3$ of the LAD was $8.99 \mathrm{~mm}$, the middle $1 / 3$ of the artery was $6.37 \mathrm{~mm}$, and the distal $1 / 3$ was $3.27 \mathrm{~mm}$. In end-inspiration (IP) compared to end-expiration the mean posterior displacements of the proximal 1/3 of the LAD was $2.08 \mathrm{~mm}$, the middle $1 / 3$ of the artery was $0.91 \mathrm{~mm}$, and the distal $1 / 3$ was $0.97 \mathrm{~mm}$. Mean doses to the LAD using tangential treatment fields and a prescribed dose of 50.4 Gy were 11.32 Gy in EP, 8.98 Gy in IP, and 3.50 Gy in DIBH. Mean doses to the LV were 2.38 Gy in EP, $2.31 \mathrm{~Gy}$ in IP, and 1.24 Gy in DIBH. In conclusion, inspiration and especially DIBH, cause a displacement of the origin and proximal 2/3 of the LAD away from the chest wall, resulting in sparing of the most critical segment of the artery during tangential radiotherapy.
\end{abstract}

Keywords: Breast; Radiotherapy; Deep-Inspiration Breath Hold (DIBH); Respiratory Gating; Left Anterior Descending Artery (LAD); Left Ventricle (LV)

\section{Introduction}

Several prospective randomized trials have demonstrated a significant reduction in locoregional recurrence with the addition of radiotherapy after breast-conserving surgery for invasive or in-situ carcinoma or after mastectomy in select patients with invasive carcinoma [1]. This improvement in local control results in improved breast cancer specific mortality and overall survival [2]. Despite these improvements in breast cancer outcomes, however, there are known long-term cardiac sequelae, including increased coronary artery disease and myocardial infarctions, in patients who have received left-sided compared to right-sided radiation treatment $[3,4]$. Newer radiotherapy treatment planning techniques have attempted to address this concern with modulation of dose to the heart using conformal radiotherapy or intensity-modulated radiotherapy, [5,6] placement of heart blocks, or

"Corresponding author using breathing adapted radiotherapy [7,8].

Investigators in Denmark have demonstrated that cardiac dose and dose to the left anterior descending artery (LAD) are substantially reduced during end-inspiration and deep inspiration breath hold (DIBH) compared to end-expiration when treating patients for left-sided breast cancer with inclusion of the upper internal mammary nodes (IMN) [8]. Using a commercially available respiratory gating device (Real-Time Position Management $(\mathrm{RPM})^{\mathrm{TM}}$ Medical Systems (Varian Medical Systems, Palo Alto, CA) these investigators suggest that respiretory gating during radiotherapy in addition to modern treatment planning techniques may further reduce the risk of long-term cardiac toxicity in patients undergoing radiotherapy for left-sided breast cancers.

These results from Denmark are striking when considering left-sided radiotherapy treatment fields that include the IMNs, which can substantially increase radiation dose to the lung and heart compared to fields that do 
not specifically target the IMNs. While there is still much debate as to the impact of internal mammary nodal irradiation on overall outcome, our current practice has been to treat the intact breast and chest wall without specific treatment of the clinically uninvolved IMNs. One could hypothesize that our treatment method in combination with respiratory gating or DIBH would even further reduce dose to the heart and the LAD as the tangential fields would be lees deep when IMN's are excluded. Currently there are no published data comparing the dosimetric impact of using DIBH versus end inspiration (IP) and end-expiration (EP) gated radiotherapy on the heart and the LAD for this treatment method.

Thus, in this study we evaluated the impact of respiratory gating and DIBH in combination with modern 3D treatment planning techniques for left sided breast cancer patients for which IMN's were not targeted. In addition, with known data that the volume of left ventricle (LV) in the treatment field results in increased myocardial perfusion defects [9] and that a significant number of treated patients have lesions identified on cardiac catheterization in the distribution of the LAD, [10] this study also evaluates whether displacement of the heart during inspiration and DIBH would result specifically in decreased volume of LV and LAD within the radiation field, as this could have the most critical impact on reducing late cardiac morbidity and mortality.

\section{Materials and Methods}

\subsection{Study Population and Simulation}

The study population consisted of 11 left-sided breast cancer patients referred for postoperative RT following mastectomy (3), mastectomy with immediate tissue expander placement for subsequent reconstruction (1), or breast-conserving surgery (7). Patients were immobilized in the supine position with the arms above the head using a custom formed binary foam mold (Alpha Cradle, Smither Products Inc., North Canton, OH). Radiopaque markers were clinically placed to delineate the breast or chest wall borders. 4-D CT scans were acquired on a GE Discovery PET/CT Scanner (General Electric Medical Systems, Waukesha, WI) equipped with the Real-Time Position Management system (RPM, Varian Medical Systems, Palo Alto, CA) for monitoring the patients' breathing, using previously describe acquisition techniques [11]. Slice thickness was $2.5 \mathrm{~mm}$. Ten reconstructed phase bins were used, yielding 10 full field volumetric image datasets per respiratory cycle for each patient from which IP and EP phases were extracted. Image processing was performed on an Advantage Workstation 4.1 with Advantage 4-D CT software (GE Medical Systems, Waukesha, WI). Static images during deep inspiration breath hold (DIBH) were also acquired. CT images were transferred to a Varian Eclipse treatment planning workstation (Varian Medical Systems, Palo Alto, CA).

\subsection{Treatment Planning}

Normal structures, including the LV (wall and cavity) and LAD, were contoured on each CT slice for each of the 3 respiratory phases analyzed (IP, EP, and DIBH). The LAD is contoured from its origin off the left main coronary artery and extending $4.5 \mathrm{~cm}$ along the length of the vessel. The vertical (anterior-posterior) distance between the LAD and the chest wall was measured on 13 consecutive CT slices per phase and per patient. Conventional 3D treatment plans for chest wall (4) or intact breast (7) radiotherapy were generated for each patient at each respiratory phase (IP, EP, DIBH) using the Varian Eclipse treatment planning system with a prescribed dose of 50.4 Gy in 28 fractions using standard coplanar tangent fields. Wedges were used to modulate the dose and to restrict the maximum dose to $<110 \%$ of the prescribed dose. The central lung distance was limited to $<$ or $=2$ $\mathrm{cm}$ for all patients as per institutional practice. Dosevolume histogram analysis was performed for each treatment plan. Measurements of chest wall motion during each of the respiratory phases were made at the level of the fifth thoracic vertebra in the anterior-posterior direction. The LAD was divided into the proximal third, middle third, and distal third, and motion was assessed for each division. Doses delivered to the LAD and LV were analyzed and compared between the 3 respiratory phases for each patient.

\section{Results}

\subsection{Patient Characteristics}

The median age of the 11 patients with left-sided breast cancers included in this dosimetric and respiratory analysis is 57 (range $=35$ - 66). Seven patients (64\%) were treated with breast-conserving surgery followed by whole breast radiotherapy and 4 (36\%) underwent mastectomy and chest wall and nodal radiotherapy that did not specifically target the IMNs.

\subsection{Motion of the Chest Wall and Left Anterior Descending Artery}

During free breathing, we found that all patients showed regular respiratory motion and that there was a negligible difference in chest wall expansion during free breathing as measured between normal end-inspiration and endexpiration (mean displacement $=0.0 \mathrm{~mm}+/-3.9 \mathrm{~mm}$ ). During DIBH, however, the chest wall was much more 
dramatically expanded in comparison to both normal end-inspiration (10.0 mm $+/-4.9 \mathrm{~mm})$ and normal end-expiration $(9.9 \mathrm{~mm}+/-4.4 \mathrm{~mm})$. In addition, the cardiac contour elongated and was displaced caudally and posteriorly in all patients during inspiration and DIBH compared to expiration. The cardiac shifts were associated with a displacement of the LAD away from the chest wall that was notable during normal inspiration compared to expiration but was greater with DIBH (Figure 1).

The movement of the LAD, however, was not uniform throughout the length of the artery. The proximal third of the LAD was displaced the greatest distance from the chest wall during both normal inspiration and DIBH compared to expiration, while the middle and distal third of the artery were only minimally displaced during normal inspiration (Table 1).

During IP, the mean posterior displacement of the LAD compared to EP was $2.1+/-4.0 \mathrm{~mm}$ for the proximal $1 / 3$ of the artery, $0.9 \mathrm{~mm}+/-3.3 \mathrm{~mm}$ for the middle $1 / 3$, and $1.0 \mathrm{~mm}+/-1.5 \mathrm{~mm}$ for the distal $1 / 3$. In DIBH, the mean posterior displacement of the LAD compared to EP was $9.0 \mathrm{~mm}+/-3.8 \mathrm{~mm}$ for the proximal $1 / 3$ of the artery, $6.4 \mathrm{~mm}+/-2.9 \mathrm{~mm}$ for the middle $1 / 3$, and $3.3 \mathrm{~mm}+/-2.9 \mathrm{~mm}$ for the distal $1 / 3$. The LAD moved away from the chest wall in all patients during DIBH. During end-inspiration, however, the LAD in 4 patients unexpectedly moved closer to, rather than further from, the chest wall compared to end-expiration. In these 4 patients, there appeared to be no correlation with the type of surgery ( $n=3$ with BCT; $n=1$ with MRM), age of the patient, or body mass index ( $\mathrm{n}=3$ with BMI > 27; $\mathrm{n}=1$ with BMI $<20$ ) to explain the variable cardiac and/or diaphragmatic motion to account for this finding.

\subsection{Radiation Dose to the Left Anterior Descending Artery and the Left Ventricle}

Doses calculated for the LAD and LV during the 3 respiratory phases analyzed are given in Table 2 . Because the LAD is located anterior to the $\mathrm{LV}$, the doses received to the LAD were higher during all respiratory phases. For standard tangential treatment to the whole breast or chest wall, the mean dose to the LAD was $22.5 \%$ of the prescription dose (PD) during EP, $17.8 \%$ of the PD during IP, and $6.9 \%$ of the PD during DIBH. The mean reducetion in dose to the LAD was 2.33 Gy from EP to IP and 7.82 Gy from EP to DIBH. For the left ventricle, the mean dose was $4.7 \%$ of the PD during EP, $4.5 \%$ of the PD during IP, and $2.5 \%$ of the PD during DIBH. The mean reduction in dose to the $\mathrm{LV}$ was $0.07 \mathrm{~Gy}$ from EP to IP and 1.14 Gy from EP to DIBH.

The volumes of left ventricle receiving $5,10,20,30$, and 40 Gy are listed in Table 3 . Three of the 11 patients analyzed had minimal dose to the LV during all respiratory phases due to the posterior positioning of the heart relative to the chest wall. For the remaining 8 patients, the mean volume of $\mathrm{LV}$ receiving $5 \mathrm{~Gy}$ (V5) was $6.7 \%$ (range $2.1 \%$ - 13.1\%) in EP, 8\% (range 1.5\% - 14.1\%) in IP, and $0.7 \%$ (range $0 \%-4.5 \%$ ) in DIBH. In these patients, the median decrease in LV V5 was $0.1 \%$ (range $1.2 \%-3.0 \%$ ) from EP to IP, and $6.0 \%$ (range $1.1 \%$ $10.2 \%)$ from EP to DIBH.

\section{Discussion}

Our results show that although there is minimal chest wall motion during normal free breathing, the cardiac contour is displaced caudally and posteriorly away from the chest wall during end-inspiration and more strikingly during DIBH, allowing substantially more distance
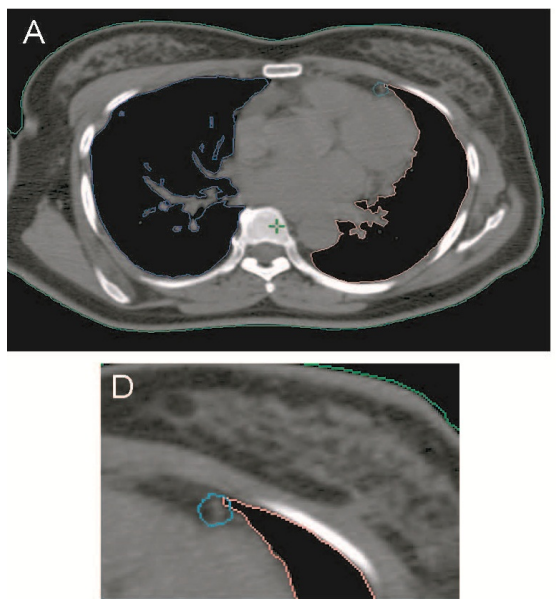
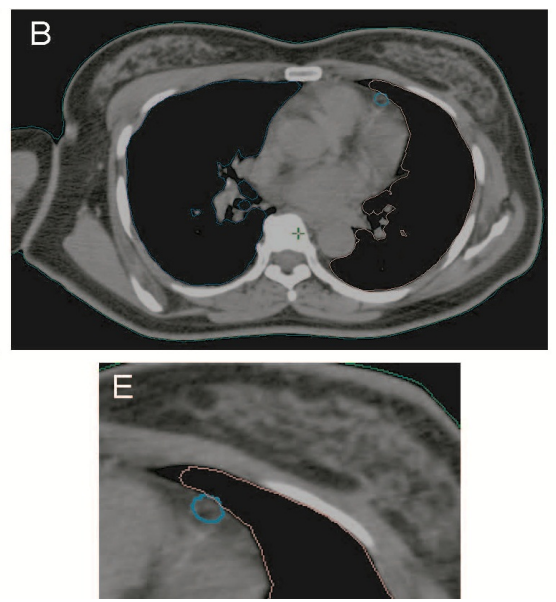
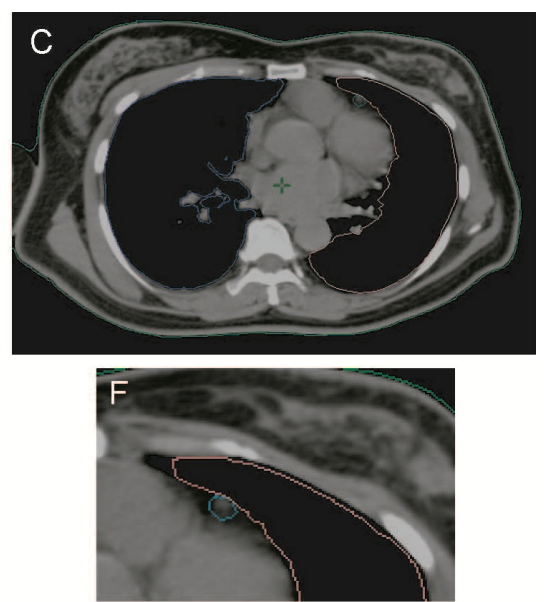

Figure 1. Position of the left-anterior descending artery (LAD) during (A) end-expiration, (B) end-inspiration, and (C) deep inspiration breath hold (DIBH). The LAD is in light blue. Lower panels are a close up view of the LAD at each respiratory phase as above (D-F). 
Table 1. Differences in displacement of the LAD from the chest wall during various respiratory phases $(\mathrm{mm})$.

\begin{tabular}{cccc}
\hline $\begin{array}{c}\text { End-inspiration vs } \\
\text { End-expiration }\end{array}$ & Mean Value & Median Value & Range \\
\hline Proximal 1/3 LAD & 2.08 & 3.00 & $-3.93-5.58$ \\
Middle 1/3 & 0.91 & 1.90 & $-7.65-5.35$ \\
Distal 1/3 & 0.97 & 1.04 & $-1.14-4.10$ \\
DIBH vs End-expiration & & & \\
Proximal 1/3 LAD & 8.99 & 8.18 & $4.98-15.95$ \\
Middle 1/3 & 6.37 & 5.98 & $2.45-11.68$ \\
Distal 1/3 & 3.27 & 3.30 & $-1.40-8.58$ \\
\hline
\end{tabular}

Table 2. Radiation dose to the LAD and LV during 3 respiratory phases $(\mathrm{Gy})$.

\begin{tabular}{cccc}
\hline LAD & Mean Value & Median Value & Range \\
\hline End-expiration & 11.32 & 9.07 & $1.52-31.40$ \\
End-inspiration & 8.98 & 8.50 & $1.47-18.18$ \\
DIBH & 3.50 & 2.74 & $1.09-13.08$ \\
LV & & & \\
End-expiration & 2.38 & 2.03 & $0.71-4.99$ \\
End-inspiration & 2.31 & 1.81 & $0.62-5.20$ \\
DIBH & 1.24 & 1.15 & $0.53-2.29$ \\
\hline
\end{tabular}

Table 3. Volume of $\mathrm{LV}$ receiving various radiation doses (\%).

\begin{tabular}{|c|c|c|c|c|c|c|}
\hline & \multicolumn{2}{|c|}{ End-expiration } & \multicolumn{2}{|c|}{ End-inspiration } & \multicolumn{2}{|c|}{ DIBH } \\
\hline & Median & Range & Median & Range & Median & Range \\
\hline \multicolumn{7}{|l|}{ LV } \\
\hline V5 & 4.5 & $0-13$ & 2.7 & $0-14$ & 0 & $0-4.5$ \\
\hline V10 & 2.1 & $0-10$ & 1 & $0-10$ & 0 & $0-3$ \\
\hline V20 & 0.8 & $0-7$ & 0.1 & $0-7$ & 0 & $0-1$ \\
\hline V30 & 0.3 & $0-6$ & 0 & $0-6$ & 0 & $0-0.5$ \\
\hline V40 & 0.04 & $0-4$ & 0 & $0-4$ & 0 & $0-0.1$ \\
\hline
\end{tabular}

between the breast/chest wall and the left ventricle and LAD. These shifts resulted in decreased dose to the LV and LAD during end-inspiration compared to end-expiration, and a substantial decrease in dose to both structures during DIBH. We also found that these differences were related to the individual patient's anatomy, with end-inspiration or DIBH having a minimal effect in those patients whose heart was positioned more posteriorly in the chest with a larger anterior-posterior separation compared to those in whom a significant percentage of the heart was located anteriorly against the chest wall, where inspiration or DIBH had a greater impact in the radiation exposure to the LV or LAD.

We also noted a non-uniform displacement of the LAD with respiratory motion. Most of the arterial motion was in the proximal two-thirds of the artery, particularly during DIBH. These findings may be particularly significant given the data from Correa et al where they noted proximal and mid-vessel LAD stenoses in 8 patients and distal stenoses in 4 [3]. In addition to improved treatment planning techniques, displacing the proximal two thirds of the LAD out of the radiation field during end-inspiration and especially DIBH may further reduce the long-term risk of coronary artery disease in these patients.

Our data are consistent with previously published data by Korreman at al. who have already demonstrated breathing adapted radiotherapy can be used to reduce the volume of irradiated heart and LAD when treating women with left-sided breast cancer when including the IMNs. They demonstrated that the median heart volume receiving $>50 \%$ of the prescribed dose could be reduced from $19.2 \%$ during free breathing to $1.9 \%$ if the patient is in deep inspiration breath hold, and the median LAD volume from $88.9 \%$ during free breathing to $3.6 \%$ for DIBH [8]. With such dramatic reductions in radiation dose, they estimated that the long-term cardiac mortality probability could potentially be reduced from $4.8 \%$ when patients are treated during free breathing to $0.1 \%$ for $\mathrm{DIBH}$ [7]. A significant difference in our study is that the $\mathrm{DIBH}$ technique significantly decreases doses to the LAD and the heart compared to end inspiration gated radiotherapy, which is not as pronounced in the Danish study.

Although breathing adapted radiotherapy and 3D treatment planning techniques such as the partially wide tan- 
gent fields [5] have been shown to reduce cardiac exposure when treating left-sided lesions when including the IMNs, data from Correa et al demonstrated that even patients who did not receive targeted treatment of the IMNs are at risk of long-term cardiac toxicity [3]. In the current study, we evaluated whether there is any additional benefit with breathing adapted radiotherapy in the setting of left-sided radiotherapy without treatment of the IMNs. That is, with good treatment planning to restrict $<$ $5 \%$ of the left ventricle within the radiation field and assuming careful daily set up to reduce set-up error and increased cardiac exposure as described by Evans et al [10], are respiratory maneuvers such as DIBH necessary?

Long-term follow up data from meta-analyses have demonstrated an excess of non-breast cancer deaths and cardiac mortality in women who undergo radiotherapy as part of their breast cancer treatment [1]. Furthermore, several epidemiologic studies report that the increased rates of radiation-associated mortality from heart disease are greater for women treated for left-sided cancers compared to right-sided lesions [12,13]. While the addition of radiotherapy has been shown to impact the rate of local recurrence and improve overall survival, [2] reducing the long-term cardiac sequelae for patients receiving radiotherapy for left-sided lesions is an important goal, particularly in an era where obesity and cardiac disease are becoming a nationwide problem and cardiotoxic agents, such as doxorubicin and trastuzumab, are currently used as standard adjuvant therapy for early-stage breast cancer.

Investigators at Duke University were among the first to directly correlate the volume of irradiated left ventricle with cardiac perfusion defects. Their early studies demonstrated that radiotherapy to the left chest wall or intact breast resulted in new perfusion defects in $60 \%$ of women evaluated 6 months after treatment [14]. Not unexpectedly these changes were dose- and volume-dependent, with increasing perfusion defects seen in regions of the left ventricle that received higher radiation doses, and increased risk in those patients where $>5 \%$ of the left ventricle was in the radiotherapy treatment field $[9,14]$. Follow up studies reported that these perfusion defects are persistent for up to 24 months, and are associated with regional wall motion abnormalities, subtle reductions in left-ventricular ejection fraction, and increased reporting of chest pain $[9,15]$. Interestingly, the increased perfusion defects were seen primarily in the distribution of the left-anterior descending artery in the regions of the irradiated myocardium, with no increases in perfusion defects in the left circumflex or right coronary artery distribution [16]. In addition to volume of LV in the treatment field, they also found that an elevated body mass index also increased the risk for perfusion defects, which was thought to possibly be secondary to an increased frequency of deep set-up errors in overweight patients compared with those with a lower BMI [10]. With the results of these studies, the investigators proposed microvascular damage as the mechanism of radiation-induced cardiac disease, with the suggestion that minimizing the volume of LV in the treatment field and reducing patient set-up errors may limit long-term cardiac toxicities in patients treated for left-sided lesions.

Investigators at the University of Pennsylvania have also demonstrated that patients treated with radiotherapy for left-sided lesions compared to right-sided had a higher rate of chest pain, coronary artery disease, and myocardial infarction with long-term follow up [4]. In addition, of 82 patients treated with conventional tangential beam RT between 1977 and 1995 who had undergone subsequent cardiac stress testing at their institution, they found that $59 \%$ of patients with left-sided lesions had abnormal stress tests compared to $8 \%$ of patients with right-sided lesions at a median follow up of 12 years after completion of radiotherapy [3]. Interestingly, 70\% of the left-sided stress test abnormalities were in the region of the left anterior descending artery and of the 13 patients with left-sided lesions who underwent cardiac catheterization, the left anterior descending artery was affected in 11 patients (85\%) and was the sole vessel affected in 8 (62\%). Only four of these 11 patients had IMN irradiation. As is pointed out by the authors, these findings are remarkable when compared to the expected distribution of coronary artery disease reported for women, where $46 \%$ are in the left anterior descending territory, $38 \%$ in the right coronary artery, and $15 \%$ in the left circumflex artery [17].

With these data supporting both a microvascular and a macrovascular etiology of radiation-induced cardiac damage for patients treated with radiotherapy for leftsided lesions, the goal in treating these patients is to reduce the volume of left ventricle that is within the radiation field as well as minimize exposure to the LAD.

Although treatment plans during DIBH demonstrated the most significant reduction in LV and LAD dose, implementation of this technique into the clinic has its challenges. As patients are instructed to take and hold a deep breath, there is variability in how deep the patient breathes at each hold. The development of a spirometer technique, such as with the Active Breathing Control (ABC) device [18] or an audio-visual screen to assist the patient in achieving a reproducible breath hold each time, is important. In addition, treatment during DIBH lengthens the overall treatment time as the radiation dose is delivered only during the breath hold. Finally, close attention to the accuracy of treatment is imperative when treating a third field to the supraclavicular region so as to 
avoid field overlaps.

In conclusion, treatment of left-sided breast cancers during end-inspiration or DIBH can substantially reduce the radiation dose to the left ventricle and left-anterior descending artery compared to treatment during endexpiration. In particular, inspiration and especially DIBH cause a displacement of the origin and proximal $2 / 3$ of the LAD away from the chest wall, resulting in the potential to decrease radiation dose to the most critical segment of the artery during tangent field radiotherapy. In order to minimize a patient's long-term risk of coronary artery disease and cardiac morbidity, we recommend tailoring the delivery of left-sided breast radiotherapy to the patient's anatomy, using a respiratorygated CT for evaluation of heart position at baseline and the amount of displacement with inspiration or DIBH. In addition to improved 3D treatment planning techniques, even patients receiving radiotherapy for left-sided breast cancers without specific targeting of the IMNs may benefit from the use of respiratory-gated treatments.

\section{Conflict of Interest}

We certify that regarding this paper, no actual or potential conflicts of interests exist; the work is original, has not been accepted for publication nor is concurrently under consideration elsewhere, and will not be published elsewhere without the permission of the Editor. All the authors have contributed directly to the planning, execution or analysis of the work reported or to the writing of the paper.

\section{REFERENCES}

[1] Early Breast Cancer Trialists’ Collaborative Group, “Effects of Radiotherapy and Surgery in Early Breast Cancer. An Overview of the Randomized Trials,” The New England Journal of Medicine, Vol. 333, No. 22, 1995, pp. 1444-1455. doi:10.1056/NEJM199511303332202

[2] M. Clarke, R. Collins, S. Darby, C. Davies, P. Elphinstone, et al., "Effects of Radiotherapy and of Differences in the Extent of Surgery for Early Breast Cancer on Local Recurrence and 15-Year Survival: An Overview of the Randomised Trials,” Lancet, Vol. 366, No. 9503, 2005, pp. 2087-2106.

[3] C. R. Correa, H. I. Litt, W. T. Hwang, V. A. Ferrari, L. J. Solin, et al., "Coronary Artery Findings after Left-Sided Compared with Right-Sided Radiation Treatment for EarlyStage Breast Cancer,” Journal of Clinical Oncology, Vol. 25, No. 21, 2007, pp. 3031-3037. doi:10.1200/JCO.2006.08.6595

[4] E. E. Harris, C. Correa, W. T. Hwang, J. Liao, H. I. Litt, et al., "Late Cardiac Mortality and Morbidity in EarlyStage Breast Cancer Patients after Breast-Conservation Treatment," Journal of Clinical Oncology, Vol. 24, No. 25, 2006, pp. 4100-4106.

\section{doi:10.1200/JCO.2005.05.1037}

[5] E. A. Krueger, M. J. Schipper, T. Koelling, R. B. Marsh, J. B. Butler, et al., "Cardiac Chamber and Coronary Artery Doses Associated with Postmastectomy Radiotherapy Techniques to the Chest Wall and Regional Nodes," International Journal of Radiation Oncology, Biology and Physics, Vol. 60, No. 4, 2004, pp. 1195-1203. doi:10.1016/j.ijrobp.2004.04.026

[6] V. M. Remouchamps, F. A. Vicini, M. B. Sharpe, L. L. Kestin, A. A. Martinez, et al., "Significant Reductions in Heart and Lung Doses Using Deep Inspiration Breath Hold with Active Breathing Control and IntensityModulated Radiation Therapy for Patients Treated with Locoregional Breast Irradiation,” International Journal of Radiation Oncology, Biology and Physics, Vol. 55, No. 2, 2003, pp. 392-406. doi:10.1016/S0360-3016(02)04143-3

[7] S. S. Korreman, A. N. Pedersen, L. R. Aarup, T. J. Nottrup, L. Specht, et al., "Reduction of Cardiac and Pulmonary Complication Probabilities after Breathing Adapted Radiotherapy for Breast Cancer,” International Journal of Radiation Oncology, Biology and Physics, Vol. 65, No. 5, 2006, pp. 1375-1380. doi:10.1016/j.ijrobp.2006.03.046

[8] S. S. Korreman, A. N. Pedersen, T. J. Nottrup, L. Specht, and H. Nystrom, "Breathing Adapted Radiotherapy for Breast Cancer: Comparison of Free Breathing Gating with the Breath-Hold Technique,” Radiotherapy \& Oncology, Vol. 76, No. 3, 2005, pp. 311-318. doi:10.1016/j.radonc.2005.07.009

[9] L. B. Marks, X. Yu, R. G. Prosnitz, S. M. Zhou, P. H. Hardenbergh, et al., "The Incidence and Functional Consequences of RT-Associated Cardiac Perfusion Defects," International Journal of Radiation Oncology, Biology and Physics, Vol. 63, No. 1, 2005, pp. 214-223. doi:10.1016/j.ijrobp.2005.01.029

[10] E. S. Evans, R. G. Prosnitz, X. Yu, S. M. Zhou, D. R. Hollis, et al., "Impact of Patient-Specific Factors, Irradiated Left Ventricular Volume, and Treatment Set-Up Errors on the Development of Myocardial Perfusion Defects after Radiation Therapy for Left-Sided Breast Cancer," International Journal of Radiation Oncology, Biology and Physics, Vol. 66, No. 4, 2006, pp. 1125-1134. doi:10.1016/j.ijrobp.2006.06.025

[11] T. Pan, T. Y. Lee, E. Rietzel and G. T. Chen, “4D-CT Imaging of a Volume Influenced by Respiratory Motion on Multi-Slice CT," Medical Physics, Vol. 31, No. 2, 2004, pp. 333-340. doi:10.1118/1.1639993

[12] S. H. Giordano, Y. F. Kuo, J. L. Freeman, T. A. Buchholz, G. N. Hortobagyi, et al., "Risk of Cardiac Death after Adjuvant Radiotherapy for Breast Cancer," Journal of the National Cancer Institute, Vol. 97, No. 6, 2005, pp. 419424. doi:10.1093/jnci/dji067

[13] L. F. Paszat, W. J. Mackillop, P. A. Groome, K. Schulze and E. Holowaty, "Mortality from Myocardial Infarction following Postlumpectomy Radiotherapy for Breast Cancer: A Population-Based Study in Ontario, Canada," International Journal of Radiation Oncology, Biology and Physics, Vol. 43, No. 4, 1999, pp. 755-762. 
doi:10.1016/S0360-3016(98)00412-X

[14] P. H. Hardenbergh, M. T. Munley, G. C. Bentel, R. Kedem, S. Borges-Neto, et al., "Cardiac Perfusion Changes in Patients Treated for Breast Cancer with Radiation Therapy and Doxorubicin: Preliminary Results," International Journal of Radiation Oncology, Biology and Physics, Vol. 49, No. 4, 2001, pp. 1023-1028. doi:10.1016/S0360-3016(00)01531-5

[15] X. Yu, R. R. Prosnitz, S. Zhou, P. H. Hardenberg, A. Tisch, et al., "Symptomatic Cardiac Events following Radiation Therapy for Left-Sided Breast Cancer: Possible Association with Radiation Therapy-Induced Changes in Regional Perfusion," Clinical Breast Cancer, Vol. 4, No. 3, 2003, pp. 193-197.

[16] P. A. Lind, R. Pagnanelli, L. B. Marks, S. Borges-Neto, C. $\mathrm{Hu}$, et al., "Myocardial Perfusion Changes in Patients Irradiated for Left-Sided Breast Cancer and Correlation with Coronary Artery Distribution,” International Journal of Radiation Oncology, Biology and Physics, Vol. 55, No. 4, 2003, pp. 914-920. doi:10.1016/S0360-3016(02)04156-1

[17] K. S. I. J. Campbell, J. S. Douglas Jr. and J. M. Bradford, "Prevalence and Distribution of Disease in Patients Catheterized for Suspected Coronary Disease," McGrawHill, New York, 1985.

[18] V. M. Remouchamps, N. Letts, F. A. Vicini, M. B. Sharpe, L. L. Kestin, et al., "Initial Clinical Experience with Moderate Deep-Inspiration Breath Hold Using an Active Breathing Control Device in the Treatment of Patients with Left-Sided Breast Cancer Using External Beam Radiation Therapy," International Journal of Radiation Oncology, Biology and Physics, Vol. 56, No. 3, 2003, pp. 704-715. doi:10.1016/S0360-3016(03)00010-5

\begin{abstract}
Abbreviations
DIBH: deep-inspiration breath hold; LAD: left anterior descending artery; LV: left ventricle; EP: end-expiration; IP: end-inspiration; IMN: internal mammary nodes.
\end{abstract}

